\title{
Immunolocalization of von Willebrand Factor and Vascular Endothelial Growth Factor during Follicular Atresia in the Swamp Buffalo Ovary
}

\author{
Jun Babaan FERANIL ${ }^{1)}$, Naoki ISOBE ${ }^{2)}$ and Toshihiko NAKAO ${ }^{3)}$ \\ 1) Laboratory of Animal Science, Graduate School for International Development and \\ Cooperation, Hiroshima University, 1-5-1 Kagamiyama, Higashi-Hiroshima 739-8529, \\ ${ }^{2)}$ Graduate School of Biosphere Science, Hiroshima University, 1-4-4 Kagamiyama, Higashi- \\ Hiroshima 739-8528, ${ }^{3)}$ Laboratory of Theriogenology, Department of Veterinary Medicine, \\ Faculty of Agriculture, Yamaguchi University, 1677-1 Yoshida, Yamaguchi 753-8515, Japan
}

\begin{abstract}
The aim of this study was to investigate the distribution pattern of von Willebrand factor (vWF) and vascular endothelial growth factor (VEGF) in the healthy antral and atretic follicles of Philippine swamp buffaloes (SB) in comparison with Holstein-Friesian cows (HF). Paraffin sections of healthy follicles and atretic follicles at various stages were immunostained with vWF antibody and VEGF antibody. The density of vWF-positive capillary vessels in the theca interna significantly increased as atresia progressed in SB, whereas the density significantly decreased in late atretic follicles compared with advanced ones in HF. On the other hand, the area of vWF-positive capillary vessels in the theca interna significantly increased as atresia progressed in both SB and HF. Immunoreactions of VEGF in the granulosa cells (in all follicle types) were observed in both SB and HF. In the granulosa layer, a reduction in the VEGF immunoreaction was noted as follicles progressed from healthy to advanced atretic follicles in both animals. Granulosa cells (in both SB and HF) showed a higher immunopositive staining than theca cells. In the theca interna, VEGF immunostaining diminished as follicles progressed to the late atretic follicles in both animals. These results indicate that during atresia, changes of vWF expression are the opposite of VEGF expression in SB. Both vWF and VEGF are suggested to be associated with follicular atresia in SB.
\end{abstract}

Key words: Atretic follicles, Philippine swamp buffaloes, Thecal microvasculature, Vascular endothelial growth factor, von Willebrand factor

(J. Reprod. Dev. 51: 419-426, 2005)

$\mathbf{T}_{\mathrm{s}}^{\mathrm{t}}$ he von Willebrand factor (vWF), a multimeric glycoprotein, is present in the plasma and subendothelium [1] and mediates adhesion of platelets to the subendothelium at the sites of vascular injury and disorder $[2,3]$. The vWF is synthesized by endothelial cells (EC) and megakaryocytes, and then stored in EC (WeibelPalade bodies) and platelets ( $\alpha$-granules) [4-6]. It is

Accepted for publication: March 29, 2005

Published online: April 22, 2005

Correspondence: N. Isobe (e-mail: niso@hiroshima-u.ac.jp) known that increased expression of vWF in blood vessel endothelial cells is associated with endothelial damage $[7,8]$, blood vessel maturation [9] and regression [10]. It has been reported that the regression process in atretic follicles coincides with an increased expression of vWF [11, 12].

Conversely, the vascular endothelial growth factor (VEGF) is considered to be a major angiogenic factor in the ovary [13], and its production declines in atretic follicles [14]. VEGF plays a crucial role in endothelial cell proliferation 
[15], chemotaxis [16], and protection from apoptosis [17-19]. VEGF exerts its cellular effects through interaction with its tyrosine kinase receptors Flt-1 (VEGF-R1) and Flk-1/KDR (VEGFR2), which bind the ligand with high affinity [20].

The relationship between thecal vasculature distribution and follicular development or atresia has been shown in domestic animals: cattle [21, 22], sheep [23, 24], and pigs [24, 25]. However, literature concerning the vasculature surrounding follicles in the buffalo is limited, except for a recent study reporting dynamic changes (using Bandeiraea simplicifolia-I lectin) in the ovarian thecal vasculature of the swamp buffalo associated with follicular atresia [26]. We hypothesized that vWF expression will tend to increase while VEGF expression will tend to decrease as follicular atresia progresses in the buffalo ovary. Hence, the aim of this preliminary study was to determine the maturity (aging) of microvessels in the theca layers during the atretic process in the buffalo. For this purpose, the distribution of vWF was compared among types of follicle and between buffaloes and cattle. We also investigated the involvement of microvessel maturity in follicular VEGF expression.

\section{Materials and Methods}

\section{Collection and preparation of tissue}

Fifteen ovaries from healthy, non-pregnant swamp buffaloes (SB) and ten ovaries from Holstein-Friesian cows (HF) were collected from slaughterhouses in the Philippines and Japan, respectively. The estimated age of SB was 5-9 years, determined by dental examination [27]. Blood samples were also collected from SB before slaughter for determination of plasma progesterone level [28-30] to estimate whether the animals were in the luteal phase $(>1 \mathrm{ng} / \mathrm{ml})$ or not. However, age and progesterone concentration of HF were not investigated due to restrictions in the slaughterhouse. No macroscopic abnormalities were observed in the reproductive tracts of animals. Ovaries were immediately fixed in $10 \%$ formalin in phosphate buffered saline (PBS, pH 7.4) and processed for paraffin sections (3- $\mu \mathrm{m}$ thick), which were air dried on slides treated with 3aminopropyltriethoxysilane (Sigma-Aldrich Chemical Co., St. Louis, MO, USA) for the vWF immunostaining or on MAS coated slides
(Matsunami Glass Industries Ltd., Kishiwada, Japan) for immunostaining of VEGF.

\section{Immunohistochemistry}

After deparaffinization with xylene, the sections were rehydrated through a series of graded volumes of ethanol in distilled water (100, 95, 90, 80 , and $70 \%$; 15 sec each) and washed in PBS for 5 min. Antigen retrieval for $\mathrm{vWF}$ was done by incubating sections with $20 \mu \mathrm{g} / \mathrm{ml}$ proteinase $\mathrm{K}$ (Sigma) in $10 \mathrm{mM}$ Tris- $\mathrm{HCl}, \mathrm{pH} 7.4$, at $35 \mathrm{C}$ for 20 min in a humidified chamber. For VEGF, antigen retrieval was conducted by heating sections in a solution of $10 \mathrm{mM}$ sodium citrate at $121 \mathrm{C}$ for $1 \mathrm{~min}$ followed by cooling for $20 \mathrm{~min}$ at room temperature. The sections were then incubated for 10 min with $1 \%$ hydrogen peroxide to remove endogenous peroxidase activity. After washing with PBS (2 times), the sections were incubated with normal goat serum for blocking $(1: 1,200$ dilution) for $30 \mathrm{~min}$. For vWF, sections were incubated with anti-human vWF antibody (reacts specifically with the cytoplasm of endothelial cells; rabbit monoclonal; 1:750 dilution; F3520, Sigma) for $30 \mathrm{~min}$ at room temperature. For VEGF, sections were incubated overnight at $4 \mathrm{C}$ with anti-human VEGF antibody (reacts specifically with the 165, 189, and 121 amino acid splice variants of VEGF; rabbit polyclonal; 1:200 dilution; Santa Cruz Biotechnology, Inc., Santa Cruz, CA, USA). Control slides were prepared in an identical manner, except that the first antibody was replaced with normal 1 $\mu \mathrm{g} / \mathrm{ml}$ rabbit $\mathrm{IgG}$. After washing with PBS twice, immunoreactions were detected by a MAX (PO) MULTI kit (Nichirei, Tokyo, Japan) according to the manufacturer's protocol. The sections were incubated with a second antibody conjugated with peroxidase and an amino acid polymer for $30 \mathrm{~min}$ at room temperature. Then, they were incubated with substrate solution consisting of $0.2 \mathrm{mg} / \mathrm{ml}$ 3'3 diaminobenzidine- $4 \mathrm{HCl}$ and $0.005 \% \mathrm{H}_{2} \mathrm{O}_{2}$ in $0.05 \mathrm{M}$ Tris- $\mathrm{HCl}$ ( $\mathrm{pH}$ 7.5). The slides were rinsed with water and counterstained with hematoxylin. After washing with running tap water, the slides were dehydrated, cleared, and covered.

\section{Classification of follicles}

Follicles were histologically classified into healthy, early atretic, advanced atretic, and late atretic, as previously reported [26, 31, 32]. Healthy follicles had an intact granulosa layer with a 
compact and well-organized arrangement. Atretic follicles were classified based on the criteria described by $\mathrm{Xu}$ et al. [33]. Briefly, early atretic follicles had a minimal number of pyknotic cells, degenerated cells, and/or apoptotic bodies distributed along the antral border of the granulosa layer. Advanced atretic follicles contained numerous pyknotic cells, degenerated cells, and/or apoptotic bodies in the granulosa layer, while the granulosa layer disappeared in late atretic follicles. The numbers of follicles examined, for SB and HF respectively, were as follows: healthy, 12 and 15; early atretic, 5 and 14; advanced atretic, 4 and 4; and late atretic, 7 and 6 .

\section{Analysis of vWF-positive capillary vessels and VEGF immunostaining \\ All available follicles with a diameter of 3 to 5} $\mathrm{mm}$ at the largest cross-sectional area were included in the analysis. Observation was conducted at 5 equidistant areas of the theca layer in each follicle (approximately 10,000 to 50,000 $\mu \mathrm{m}^{2}$ ). The vWF-positive capillary vessels were counted using an image analyzer with a computer system (Mac Aspect, Mitani Co., Fukui, Japan) [34, 35]. The capillary vessel area contained both endothelial cells and the vessel lumen. The capillary vessel density and capillary vessel area in the theca interna and externa were expressed as values per $20,000-\mu \mathrm{m}^{2}$ area. The density and area of follicles was expressed as the average of 5 counts. The pattern of VEGF immunostaining was graded quantitatively as follows: partial, "+/-"; half, "+"; and whole " ++ ". Observation was conducted at 5 equidistant areas of each follicle at $400 \times$ magnification $(10 \times$ ocular lens, $40 \times$ objective lens) .

\section{Statistical analysis}

Differences in the vWF-positive capillary vessel density and area among different breeds, as well as among different follicular stages of atresia were analyzed by two-factor ANOVA, followed by Duncan's multiple range test (EXCEL Statistics Ver. 4; Esumi Co., Tokyo, Japan). Differences were considered to be significant at $\mathrm{P}<0.05$.

\section{Results}

Immunoassay of SB plasma samples showed that $60 \%$ of the animals were in the luteal phase (progesterone level above $1 \mathrm{ng} / \mathrm{ml}$ ). Due to a limited number of follicles available for examination, comparison of immunolocalization between animals with a progesterone level of $<1$ and $>1 \mathrm{ng} / \mathrm{ml}$ was not performed. Likewise, the frequency distribution of the different follicle sizes in the different follicle types was not investigated.

In both SB and HF, the endothelial cells of the thecal capillary vessels of atretic follicles showed a greater affinity to anti-vWF antibody than healthy follicles (Fig. 1). Capillary vessels without staining for vWF were observed in the healthy follicles (Fig $1 \mathrm{~A}$ and E). Atretic follicles also showed pyknosis of granulosa cells, and apoptotic body-like debris were present, indicating follicular atresia. Control slides showed a negative reaction, confirming the specificity of the antibody used in this study (pictures not shown).

In the theca interna, SB showed a significant increase in the density of vWF-positive capillary vessels as follicles progressed from healthy to late atretic follicles, while HF showed a plateau in early and advanced atretic follicles, which significantly declined as they progressed to the late atretic follicles (Fig. 2A). There were significant differences in density of vWF-positive capillary vessels in advanced and late atretic follicles between SB and HF. However, the area of vWFpositive capillary vessels significantly increased as atresia progressed in both breeds (Fig. 2B).

In the theca externa, density of vWF-positive capillary vessels significantly increased in the early atretic follicles of both breeds compared to those of healthy follicles (Fig. 2C). Moreover, late atretic follicles had a significantly higher number of vWFpositive capillary vessels than in the early atretic follicles of both SB and HF. HF late atretic follicles contained a significantly high number of vWFpositive capillary vessels as compared to SB. The area of vWF-positive capillary vessels significantly increased as follicles progressed to late atretic follicles in SB and advanced atretic follicles in HF (Fig. 2D).

Table 1 shows the distribution pattern of VEGF staining in the different stages of follicular atresia in SB and HF. A decrease in immunopositivity (granulosa and theca interna layers) was noted as follicles progressed from healthy to atretic follicles in both breeds. Both breeds gave a positive immunoreaction for anti-VEGF antibody in all atretic stages of follicles, with the granulosa layer 


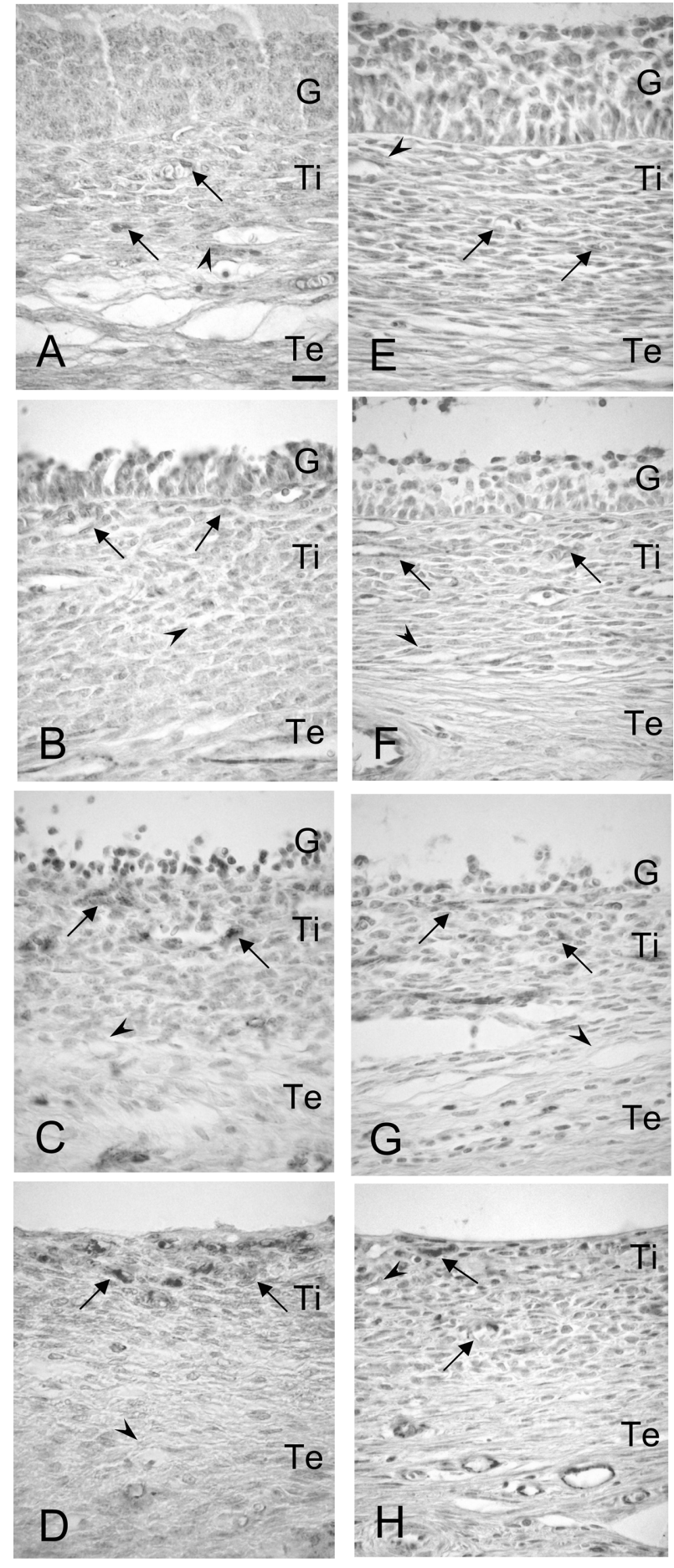

Fig. 1. The von Willebrand factor (vWF) immunostaining in the various stages of follicular atresia in buffaloes (A: healthy; B: early atretic; C: advanced atretic; D: late atretic) and Holstein-Friesian cows (E: healthy; F: early atretic; G: advanced atretic; H: late atretic). Solid arrows indicate a positive immunoreaction and arrowheads indicate a negative immunoreaction. G: Granulosa layer; Ti: Theca interna; Te: Theca externa. Scale bar $=10 \mu \mathrm{m}$. showing a better affinity for immunostaining than the theca layers (Fig. 3). In healthy follicles of both breeds, the entire granulosa layer was positively stained for VEGF antibody. However, VEGF reactivity decreased in atretic follicles, resulting in only a partial staining with VEGF antibody in advanced atretic follicles. In the theca interna of both SB and HF, half the layer was immunostained with VEGF antibody in the healthy follicles, whereas only a partial VEGF-positive layer was observed in all atretic follicles. Control slides showed a negative reaction, confirming the specificity of the antibody used in this study (data not shown).

\section{Discussion}

The preliminary findings from the present work are presented in this report. In SB, both density and area of vWF-positive capillary vessels in the theca interna increased significantly as follicles progressed from healthy to late atretic follicles. Increased expression of vWF can be associated in part to maturation and/or aging of blood vessels [9]. Therefore it is suggested that the atretic process is accompanied by an increase in matured or aged capillary vessels in the theca interna of SB. The present results for $\mathrm{SB}$ are similar to previous data we obtained from cattle [11]. The density of vWFpositive capillary vessels in the theca interna of SB increased continuously as follicles progressed from healthy to late atretic follicles, while HF showed a plateau in early and advanced atretic follicles, which significantly declined as they progressed to late atretic follicles. This suggests a difference in the changes of matured/aged capillary vessel density in the theca interna during the atretic process between SB and HF. Since the total number of capillary vessels was not altered during the atretic process [26], follicular progression to late atresia in HF reduces the density of only matured/ aged capillary vessels. In the theca interna of late atretic follicles, a significantly increased density of vWF-positive capillary vessels was attained in SB compared with HF. However, there was no difference in the total number of capillary vessels between SB and HF in the theca interna of late atretic follicles [26]. These results indicate a greater ratio of matured/aged capillary vessel in SB than HF. 

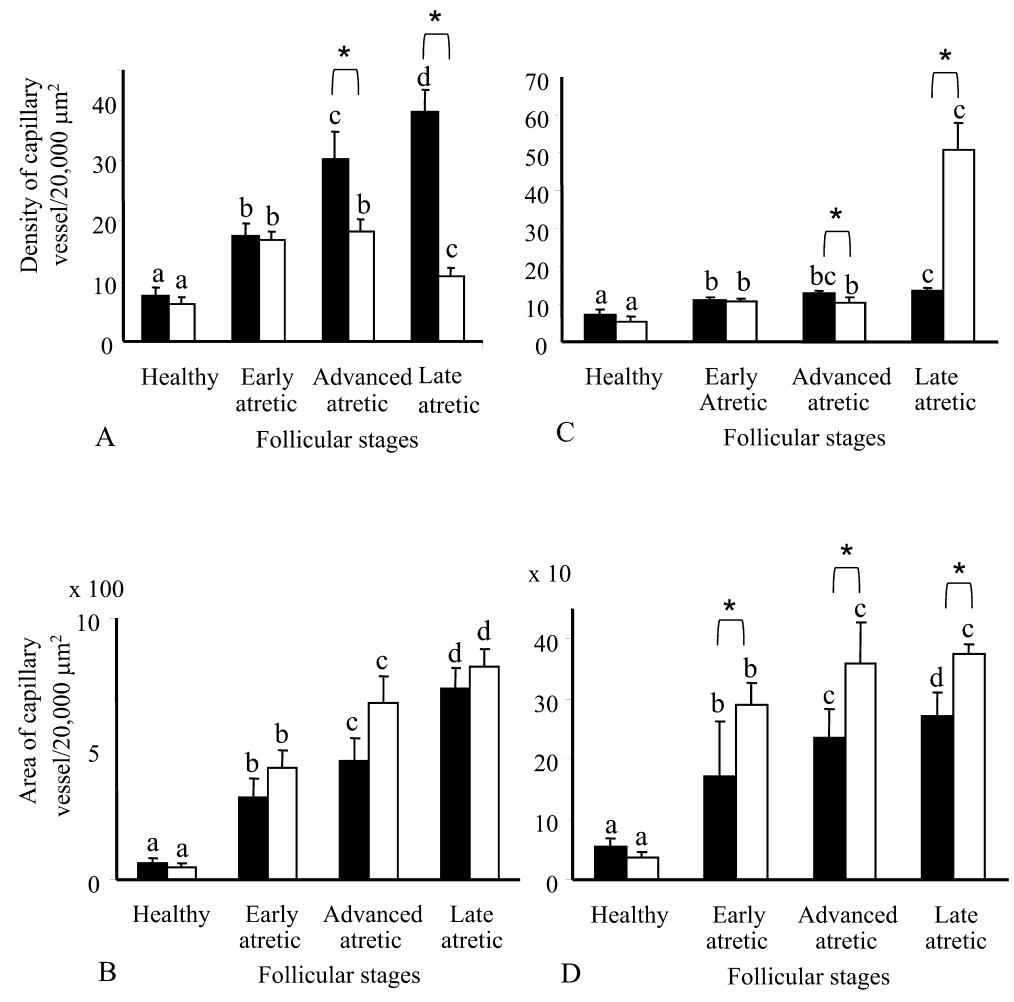

Fig. 2. Density (A and C) and area (B and D) of von Willebrand factor positivecapillary vessels in the theca interna (A and B) and externa (C and D) for various follicular stages of atresia. Bars represent the mean \pm SE. The closed and open bars represent swamp buffaloes and Holstein-Friesian cows, respectively. Means with different letters ( $a, b, c$, and d) represent a significant follicle type difference in the same breed and asterisks ${ }^{*}$ ) represent a significant breed difference in the same follicle type. The level of significance is $\mathrm{P}<0.05$.

Table 1. Distribution patterns of VEGF immunolocalization in the various stages of follicular atresia. +/partial; + half; ++ whole. (Gc: Granulosa cells, Ti: Theca interna cells)

\begin{tabular}{lcccc}
\hline & \multicolumn{2}{c}{ Swamp buffalo } & \multicolumn{2}{c}{ Holstein-Friesian } \\
Follicle type & Gc & Ti & Gc & Ti \\
\hline Healthy antral & ++ & + & ++ & + \\
Early atretic & + & $+/-$ & + & $+/-$ \\
Advanced atretic & $+/-$ & $+/-$ & $+/-$ & $+/-$ \\
Late atretic & & $+/-$ & & $+/-$ \\
\hline
\end{tabular}

In the theca externa, late atretic follicles of both SB and HF had a significantly higher density of vWF-positive capillary vessels than healthy and early atretic follicles. However, HF showed significantly higher density of vWF-positive capillary vessels than SB. This indicates a dramatic increase in the number of matured/aged capillary vessels in late atretic follicles, especially in $\mathrm{HF}$, which is quite different from SB. Since there was no difference in the total number of capillary vessels (matured and immature) in late atretic follicles between SB and HF [26], the ratio of matured capillary vessels to the total number of capillary vessels is higher in $\mathrm{HF}$ than $\mathrm{SB}$. This result seems to be opposite to the case of the theca interna, suggesting a physiological difference in capillary vessel function in late atretic follicles between SB and HF.

In the present study, we demonstrated that VEGF was localized in the granulosa and theca layers in both SB and HF. This localization was high in the granulosa of healthy follicles in SB and HF. A similar observation was noted in bovine [36] and rat [37] ovarian follicles. These previous studies also noted that an increased ligand expression resulted in enhanced capillary 

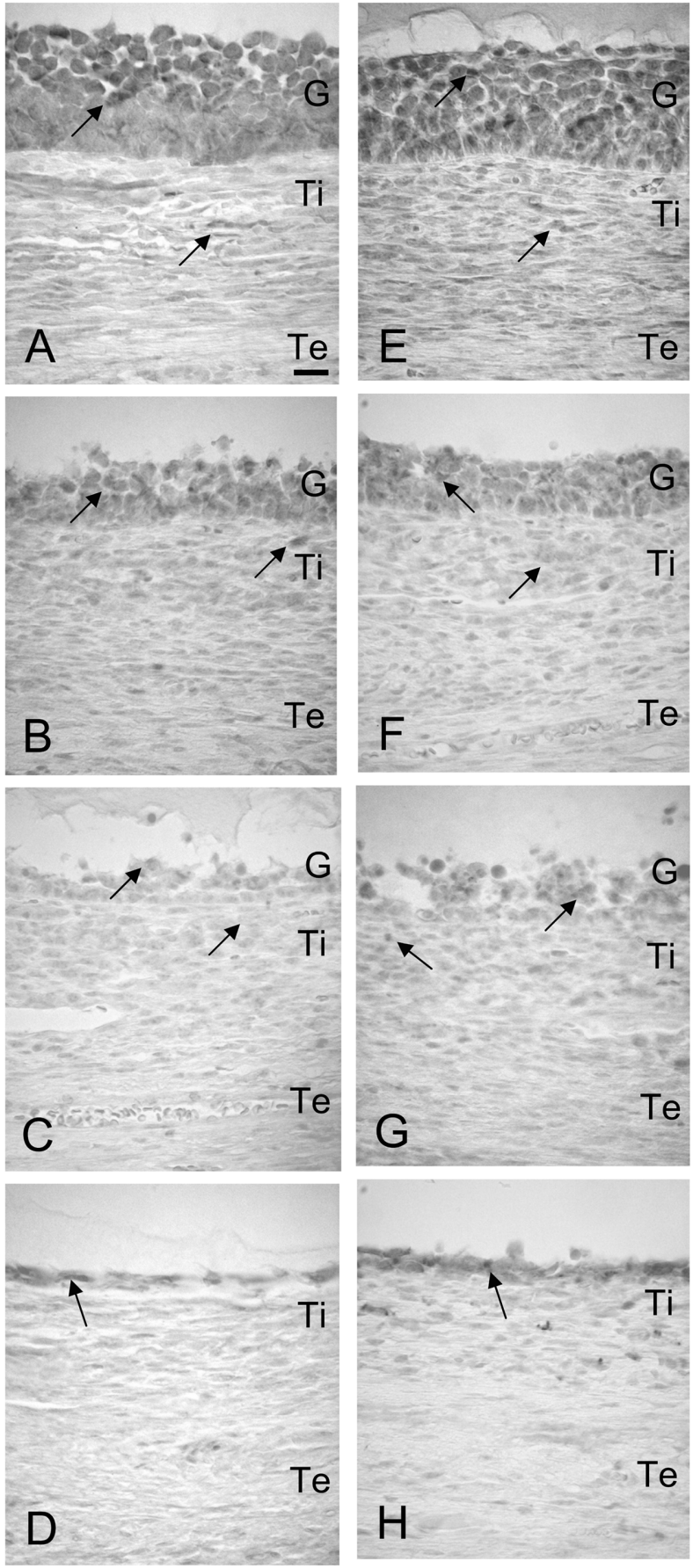

Fig. 3. Vascular endothelial growth factor (VEGF) immunostaining for the various stages of follicular atresia in buffaloes (A: healthy; B: early atretic; C: advanced atretic; D: late atretic) and HolsteinFriesian cows (E: healthy; F: early atretic; G: advanced atretic; $\mathrm{H}$ : late atretic). Arrows indicate a positive immunoreaction. G: Granulosa layer; Ti: Theca interna; Te: Theca externa. Scale bar $=10 \mu \mathrm{m}$. proliferation. Recently, it was reported that a close temporal association between VEGF expression and angiogenesis occurs in the equine corpus luteum [38]. Moreover, VEGF mRNA expression is temporally and spatially related to the proliferation of blood vessels in the ovary [39]. These findings support the concept that VEGF plays a major role as a potent regulator of angiogenesis.

Our immunohistochemical localization of VEGF suggests that the capacity of atretic follicles to produce VEGF is reduced as compared to healthy ones, which supports previous reports in other species $[14,40]$. The density and area of vWFpositive capillary vessels increased in the SB theca interna, suggesting that vWF expression is negatively correlated to VEGF expression.

We observed that alteration of VEGF expression in the theca interna was slighter (between partial and half) than in the granulosa (between partial and whole) as follicles progressed from healthy to atretic follicles in SB, as well as in HF. The formation of vasculature depends on VEGF expression [13], and vasculogenesis in the theca interna may be attributable to the VEGF secreted by granulosa cells.

In conclusion, this study demonstrates for the first time in SB ovary that matured capillary vessels (vWF-positive) increase in the process of follicular atresia. Furthermore, VEGF is expressed in the granulosa cells as well as in the theca interna of SB, and this expression tends to decrease in atretic follicles. It is suggested that vWF and VEGF are associated with follicular atresia in a converse relationship.

\section{Acknowledgements}

The first author is grateful to the Ministry of Education, Culture, Sports, Science and Technology of the Government of Japan for the scholarship granted for pursuing this research work and to Cavite State University, Philippines for logistical support during specimen collection. The present study was partly supported by a Grant-in-Aid from the Japan Society for the Promotion of Science (JSPS) to N.I. 


\section{References}

1. Meyer D, Pietu G, Fressinaud E, Girma JP. von Willebrand factor: structure and function. Mayo Clin Proc 1991; 66: 516-523.

2. Soylemezoglu O, Sultan N, Gursel T, Buyan N, Hasanoglu E. Circulating adhesion molecules ICAM-1, E-selectin, and von Willebrand factor in Henoch-Schonlein purpura. Arch Dis Childhood 1996; 75: 507-511.

3. Weiss HJ. von Willebrand factor and platelet function. Annals New York Acad Sci 1991; 614: 25-37.

4. Wagner DD, Olmsted JB, Marder VJ. Immunolocalization of von Willebrand protein in Weibel-Palade bodies of human endothelial cells. $J$ Cell Biol 1982; 95: 355-360.

5. Cramer EM, Meyer D, le Menn R, Breton-Gorius J. Eccentric localization of von Willebrand factor in an internal structure of platelet alpha-granule resembling that of Weibel-Palade bodies. Blood 1985; 66: 710-713.

6. Manucci PM. Platelet von Willebrand factor in inherited and acquired bleeding disorders. Proc Natl Acad Sci USA 1995; 92: 2428-2432.

7. Woolf AD, Wakerley G, Wallington TB, Scott DG, Dieppe PA. Factor VIII related antigen in the assessment of vasculitis. Annals Rheumatic Dis 1987; 46: 441-447.

8. Bowyer SL, Ragsdale CG, Sullivan DB. Factor VIII related antigen and childhood rheumatic diseases. $J$ Rheumatol 1989; 16: 1093-1097.

9. Augustin HG. Antiangiogenic tumour therapy: will it work? Trends Pharmacol Sci 1998; 19: 216-222.

10. Modlich U, Kaup FJ, Augustin HG. Cyclic angiogenesis and blood vessel regression in the ovary: blood vessel regression during luteolysis involves endothelial cell detachment and vessel occlusion. Lab Invest 1996; 74: 771-780.

11. Isobe N, Kawai H, Yoshimura Y, Nakao T. Changes in the localization of immunoreactive von Willebrand factor in microvascular network of bovine ovarian follicles during atresia. Anim Sci J 2001; 72: 473-482.

12. Isobe N, Yoshimura $\mathbf{Y}$, Nakao T. Distribution of immunoreactive von Willebrand factor in the microvascular network of bovine cystic follicles. Anim Sci J 2002; 73: 123-129.

13. Reynolds LP, Grazul-Bilska AT, Redmer DA. Angiogenesis in the corpus luteum. Endocrine 2000; 12: 1-9.

14. Yamamoto K, de Waard V, Fearns C, Loskutoff DJ. Tissue distribution and regulation of murine von Willebrand factor gene expression in vivo. Blood 1998; 92: 2791-2801.

15. Zeng H, Dvorak HF, Mukhopadhyay D. Vascular permeability factor (VPF)/vascular endothelial growth factor (VEGF) receptor-1 down-modulates
VPF/VEGF receptor-2-mediated endothelial cell proliferation, but not migration, through phosphatidylinositol 3-kinase-dependent pathways. J Biol Chem 2001; 276: 26969-26979.

16. Matsumoto $\mathbf{Y}$, Tanaka $\mathrm{K}$, Hirata $\mathrm{G}$, Hanada $\mathbf{M}$, Matsuda S, Shuto T, Iwamoto Y. Possible involvement of the vascular endothelial growth factor-Flt-1-focal adhesion kinase pathway in chemotaxis and the cell proliferation of osteoclast precursor cells in arthritic joints. I Immunol 2002; 168: 5824-5831.

17. Gerber HP, McMurtrey A, Kowalski J, Yan M, Keyt BA, Dixit B, Ferrara N. Vascular endothelial growth factor regulates endothelial cell survival through the phosphatidylinositol 3'-kinase/Akt signal transduction pathway. Requirement for Flk1/KDR activation. J Biol Chem 1998; 273: 3033630343.

18. Tran J, Master Z, Yu JL, Rak J, Dumont DJ, Kerbel RS. A role for surviving in chemoresistance of endothelial cells mediated by VEGF. Proc Natl Acad Sci USA 2002; 99: 4349-4354.

19. Greenaway J, Connor K, Pedersen HG, Coomber BL, Lamarre J, Petrik J. Vascular endothelial growth factor and its receptor Flk-1/KDR, are cytoprotective in the extravascular compartment of the ovarian follicle. Endocrinology 2004; 145: 28962905.

20. De Vries C, Escobedo JA, Ueno H, Houck K, Ferrara N, Williams LT. The fms-like tyrosine kinase, a receptor for vascular endothelial growth factor. Science 1992; 255: 989-991.

21. Marion GB, Gier HT, Choudary JB. Micromorphology of the bovine ovarian follicular system. J Anim Sci 1968; 27: 451-465.

22. Jiang JY, Macchiarelli G, Tsang BK, Sato E. Capillary angiogenesis and degeneration in bovine ovarian antral follicles. Reproduction 2003; 125: 211223.

23. Hay MF, Cran DG, Moor RM. Structural changes occurring during atresia in sheep ovarian follicles. Cell Tissue Res 1976; 169: 515-529.

24. Cran DG, Osborn JC, Rushton D. Thecal vasculature and oocyte maturation during follicular atresia in the sheep and pig. Reprod Nutr Dev 1983; 23: 285-292.

25. Shimizu T, Jiang JY, Sasada H, Sato E. Changes of mRNA expression of angiogenic factors and related receptors during follicular development in gilts. Biol Reprod 2002; 67: 1846-1852.

26. Feranil JB, Isobe $\mathbf{N}, \mathbf{N a k a o} \mathrm{T}$. Changes in the thecal vasculature during follicular atresia in the ovary of swamp buffalo. J Reprod Dev 2004; 50: 315-321.

27. Maala CP, Domingo R, Ducusin RJ. The anatomy of the temporary and permanent incisors of the 
carabao (Bubalus bubalis). Phil J Vet Med 1988; 25: 714.

28. Isobe N, Nakao T. Direct enzyme immunoassay of estrone sulfate in the plasma of cattle. J Reprod Dev 2002; 48: 75-78.

29. Isobe $\mathbf{N}$, Nakao T, Uehara $\mathbf{O}$, Yamashiro H, Kubota H. Plasma concentration of estrone sulfate during pregnancy in different breeds of Japanese beef cattle. J Reprod Dev 2003; 49: 369-374.

30. Isobe $\mathbf{N}, \mathbf{N a k a o} \mathbf{T}$. Direct enzyme immunoassay of progesterone in bovine plasma. Anim Sci J 2003; 74: 369-373.

31. Isobe $\mathbf{N}$, Nakao $\mathbf{T}$, Yoshimura $\mathbf{Y}$. Immunohistochemical localization of $3 \beta-$ hydroxysteroid dehydrogenase in the granulosa and theca interna layers of bovine cystic follicles. $J$ Reprod Dev 2003; 49: 227-233.

32. Feranil JB, Isobe N, Nakao T. Cell proliferation in the atretic follicles of buffalo and cattle ovary. Reprod Domest Anim 2004; 39: 405-409.

33. Xu Z, Garverick HA, Smith GW, Smith MF, Hamilton SA, Youngquist RA. Expression of follicle-stimulating hormone and luteinizing hormone receptor messenger ribonucleic acids in bovine follicles during the first follicular wave. Biol Reprod 1995; 53: 951-957.

34. Isobe N, Yoshimura Y. Localization of apoptotic cells in the cystic ovarian follicles of cows: a DNA- end labeling histochemical study. Theriogenology 2000; 53: 897-904.

35. Isobe N, Yoshimura Y. Immunocytochemical study of cell proliferation in the cystic ovarian follicles in cows. Theriogenology 2000; 54: 1159-1169.

36. Berisha B, Schams D, Kosmann M, Amselgruber $\mathbf{W}$, Einspanier R. Expression and localisation of vascular endothelial growth factor and basic fibroblast growth factor during the final growth of bovine ovarian follicles. J Endocrinol 2000; 167: 371382.

37. Koos RD. Increased expression of vascular endothelial growth/permeability factor in the rat ovary following an ovulatory gonadotropin stimulus: potential roles in follicle rupture. Biol Reprod 1995; 52: 1426-1435.

38. Al-zi'abi MO, Watson ED, Fraser HM. Angiogenesis and vascular endothelial growth factor expression in the equine corpus luteum. Reproduction 2003; 125: 259-270.

39. Phillips HS, Hains J, Leung DW, Ferrara N. Vascular endothelial growth factor is expressed in rat corpus luteum. Endocrinology 1990; 127: 965-967.

40. Celik-Ozenci C, Akkoyunlu G, Kayisli UA, Arici A, Demir R. Localization of vascular endothelial growth factor in the zona pellucida of developing ovarian follicles in the rat: a possible role in destiny of follicles. Histochem Cell Biol 2003; 120: 383-390. 